\title{
A INFLUÊNCIA DOS VALORES SUSTENTÁVEIS NA ESCOLHA DE UMA ORGANIZAÇÃO PARA SE TRABALHAR
}

\author{
Fernando Nascimento \\ Mestrando em Administração pela Universidade de São Paulo - FEA/USP \\ fernandonascimento.usp@gmail.com
}

Filippe Delarissa Barros
Mestrando em Administração pela Faculdade de Economia, Administração e Contabilidade - FEA/USP fbarros.monitoria@gmail.com

Vanessa Cuzziol Pinsky

Doutoranda em Administração pela Faculdade de Economia, Administração e Contabilidade - FEA/USP vanessa.pinsky@usp.br

\author{
Bernadete de Lourdes Marinho \\ Professora na Faculdade de Economia, Administração e Contabilidade pela Universidade de São Paulo \\ marinhoy@usp.br
}

\begin{abstract}
RESUMO
Com base na proposição de que os indivíduos valorizam organizações que apresentam um bom desempenho socioambiental, este estudo teve como objetivo avaliar a influência dos valores pessoais na escolha de uma organização para se trabalhar. Trata-se de uma pesquisa quantitativa e descritiva, utilizando-se o método survey analítico e considerando uma amostra não-probabilística, por conveniência. A coleta dos dados se deu por meio de questionário estruturado em três partes, considerando cenários de empresas, que foram avaliadas pelos respondentes em razão da disposição ou não de se trabalhar nelas, estrutura universal de valores da Teoria de Schwartz (1992) e perguntas sobre o perfil dos respondentes. Por meio da análise dos dados foi possível identificar que há indícios de que quanto maior a presença da dimensão autotranscedência - que compreende os valores universalismo e benevolência do indivíduo, segundo Teoria de Schwartz (1992) maior a importância dada ao desempenho socioambiental da empresa, e, portanto, à sustentabilidade, uma vez que o desempenho econômico das empresas é avaliado independentemente da presença ou ausência de qualquer grupo de valores. Também foi observado que indivíduos com baixa presença de valores autotranscendentes dão menos importância à remuneração. Os principais limites desse estudo foram a amostra homogênea e não estatisticamente representativa, bem como algumas limitações intrínsecas ao instrumento de coleta de dados utilizado.
\end{abstract}

Palavras-chave: Análise conjunta; Gestão de pessoas; Recursos humanos; Schwartz; Sustentabilidade; Valores pessoais.

\section{THE INFLUENCE OF SUSTAINABLE VALUE FOR CHOOSING AN ORGANIZATION TO WORK}

\begin{abstract}
The following study aims to evaluate the influence of personal values on job choice based on the proposition that individuals value organizations with good environmental and social performances. It consists of quantitative and descriptive research, using the analytical survey method, and considers a non-probabilistic convenience sample. Data collection was done through a questionnaire structured in three parts, which included scenarios of companies that were evaluated considering the intention to work or not to work in them by respondents; the universal structure of values from the Schwartz Theory (1992) and questions in relation to the profile of the respondents. Through data analysis it was possible to identify evidence that a higher presence of self-transcendence dimension - which comprehends the values of universalism and benevolence of the individual, according to Schwartz Theory (1992) - is related to a higher importance given to the environmental and social performance of the company, and, therefore, to sustainability, once companies' economical performance is evaluated regardless of the presence or absence of any group of values. It was also observed that individuals with low presence of auto-transcendent values give less attention to remuneration. The main limitations of the study were the homogeneous and not statistically representative sample, as well as few other limitations intrinsic to the data collection instrument used.
\end{abstract}

Key words: Conjoint analysis; Human resources; People management; Personal values; Schwartz; Sustainability. 


\section{INTRODUÇÃO}

O aumento da importância da sustentabilidade nos últimos anos tem pressionado as empresas a adotar práticas de negócios que consideram o equilíbrio das dimensões econômica e socioambiental nas suas atividades. A inserção da sustentabilidade na estratégia de negócio é também decorrente da pressão dos stakeholders (May, Lustosa \& Vinha, 2003) e da necessidade de redução de custos e riscos (Hart \& Milstein, 2004).

$\mathrm{Na}$ perspectiva de gestão de pessoas, a sustentabilidade também demanda novas práticas empresariais para a atração de talentos, uma atividade de grande importância para as empresas (Coff \& Kryscynski, 2011). Se uma empresa com práticas sustentáveis tem melhoria de reputação e marca (Ryder, Shahid \& Yan, 2011), e, considerando que as melhores empresas para se trabalhar (employer of choice), que são as organizações onde os principais talentos desejam trabalhar, atraem profissionais pela sua reputação e mensagem de sua marca no mercado (Sutherland, Torricelli, \& Karg, 2002), a sustentabilidade pode ser uma variável importante na escolha de uma empresa para se trabalhar. Ainda de acordo com uma pesquisa realizada pela empresa Edelman (2012), 48\% dos entrevistados em diversas localidades do mundo afirmaram que não desejam trabalhar em uma empresa que não suporte uma boa causa de interesse público, o que representa um aumento de $11 \%$ frente à mesma pesquisa de 2010, e aponta que, em pesquisa realizada com indivíduos em diversas localidades no mundo, $48 \%$ dos entrevistados afirmaram que não desejam trabalhar em uma empresa que não suporte uma boa causa, o que representa um aumento de $11 \%$ frente à mesma pesquisa de 2010. Essa é uma informação relevante principalmente em um cenário em que os recursos humanos de uma organização podem representar uma fonte de vantagem competitiva sustentável (Barney \& Wright, 1998; Chadwick \& Dabu, 2009; Coff \& Kryscynski, 2011).

Tendo em vista a importância dos recursos humanos e da atração de talentos para as organizações, e a crescente valorização da sustentabilidade como parte das estratégias de negócio e na escolha dos indivíduos por uma organização que apoie uma causa de interesse público no momento de se trabalhar, buscou-se, por meio deste estudo, avaliar se os valores pessoais orientados à sustentabilidade influenciam na escolha de uma organização para se trabalhar.

A opção dos pesquisadores por se utilizar os valores pessoais se deu porque a escolha de uma organização para se trabalhar faz parte de uma série decisões que são tomadas na procura de uma empresa (Gatewood et al, 1993), ou seja, uma ação, e, da mesma forma, os valores podem ser definidos como o critério utilizado pelas pessoas para selecionar, justificar e avaliar ações, guiando comportamentos (Allport, 1961; Rokeach, 1973; Schwartz, 1992). Além disso, foi identificado um gap da literatura, já que diversos estudos foram encontrados sobre sustentabilidade, outros sobre escolha de uma organização para se trabalhar e, por fim, estudos sobre os valores pessoais, mas nenhum articulando esses três conceitos simultaneamente.

Portanto, para essa pesquisa foi considerada a seguinte questão problema: qual a influência dos valores voltados à sustentabilidade na escolha de uma organização para se trabalhar?

O principal objetivo desse estudo foi avaliar a influência dos valores pessoais de indivíduos na escolha de uma organização para se trabalhar. Complementarmente, foram considerados os seguintes objetivos específicos: 1) validar o modelo metodológico e teórico proposto; 2) verificar se os respondentes da amostra apresentam valores pessoais orientados para sustentabilidade; 3) verificar se a importância dos critérios de desempenho da organização, remuneração e oportunidade de crescimento diferem entre indivíduos com maior presença de valores sustentáveis; 4) compreender a relação entre os perfis de valores e a importância atribuída aos critérios.

Com isso, como principais contribuições, este trabalho apresenta um instrumento de coleta desenvolvido especificamente para o estudo tendo como base um robusto referencial teórico e outros instrumentos já validados, o que permite a replicação com diferentes amostras em estudos futuros, além de se propor a compreender de que maneira os valores pessoais orientados à sustentabilidade influenciam a escolha de uma organização para se trabalhar, e quais aspectos são considerados mais importantes para os indivíduos com esses valores. 


\section{FUNDAMENTAÇÃO TEÓRICA}

Com o objetivo de embasar o modelo teórico desenvolvido para esse estudo, a revisão bibliográfica foi baseada em três pilares: estrutura universal de valores segundo a Teoria de Schwartz, sustentabilidade e escolha de uma organização para se trabalhar. A literatura escolhida converge com a proposição da pesquisa de que os valores pessoais, especialmente os valores pessoais orientados para a sustentabilidade, influenciam na decisão de um indivíduo na escolha de uma organização para se trabalhar.

\subsection{Valores pessoais}

Os valores pessoais são variáveis utilizadas em diversos estudos para compreensão do comportamento dos indivíduos e de fenômenos sociais diversos (Schwartz \& Bardi, 2001). Atualmente, as pesquisas sobre valores se fundamentam basicamente nas teorias de Schwartz (1992; 1994; 1996; 1999), por representarem conhecimentos consolidados sobre essa temática.

Schwartz e Bilsky (1987) definem valores como representações cognitivas de três tipos de necessidades humanas universais: necessidades biológicas do organismo, necessidades de interação social e necessidades socioinstitucionais, que visam o bem-estar e sobrevivência do grupo.

É importante ressaltar que não há um consenso entre os pesquisadores da temática de valores sobre a influência destes no direcionamento de comportamentos, nas escolhas e ações. Dessa forma, enquanto alguns autores consideram que os valores são capazes de guiar comportamentos e até incluem essa característica em suas definições (Allport, 1961; Rockeach, 1973), outros autores acreditam que essa influência só ocorre em casos específicos e que, por isso, não pode ser generalizada. No entanto, vários estudos empíricos, como, por exemplo, as pesquisas desenvolvidas por Rockeach (1973) e Schwartz (1996), demonstram haver relações entre os valores e o comportamento dos indivíduos.

Segundo Schwartz (1992), há um conjunto básico de valores que pode ser reconhecido em todas as sociedades. Esses valores interagem em situações diversas, possuem importância variada para o indivíduo e servem para guiar os princípios na vida de uma pessoa ou grupo. Os valores são organizados em um sistema coerente que pode ajudar a explicar atitudes, comportamentos e o processo de tomada de decisão.

Nessa perspectiva, Schwartz (1994) propõe a organização desses valores em dez categorias, denominadas por ele de tipos motivacionais, que constituem o quadro teórico a ser utilizado nessa pesquisa. Segundo Schwartz (2005), a teoria dos valores compreende uma estrutura dinâmica de relações entre os tipos motivacionais, onde as ações na busca de qualquer valor têm consequências que podem conflitar ou ser congruentes com a busca de outros valores (Schwartz, 2005).

Complementarmente, Tamayo, Lima, Marques e Martins (2001) afirmam que os tipos motivacionais interagem com os demais valores, de forma que podem se aproximar ou se opor a eles em determinadas circunstâncias. Os tipos motivacionais equivalentes, como "universalismo" e "benevolência" ou "realização" e "poder", conduzem os comportamentos para mesma direção, enquanto valores que são contraditórios ou conflitantes, como "poder" e "universalismo", motivam comportamentos em direções contrárias.

Essa composição de relações dinâmicas entre os tipos motivacionais pode ser ilustrada em uma estrutura circular, como apresentado na Figura 1. 


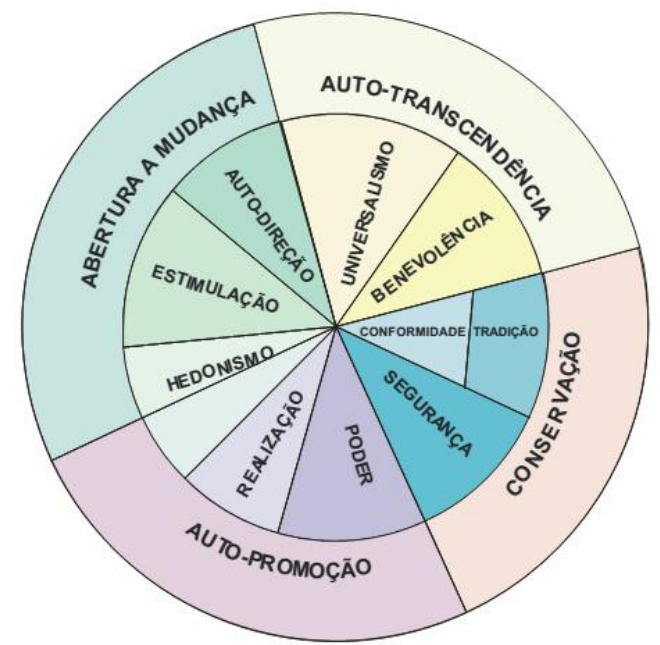

Figura 1: Estrutura universal de valores segundo a Teoria de Schwartz Fonte: Schwartz (1992; 1994), adaptado por Pato (2004)

A proposta de uma estrutura circular é possível, pois a teoria de Schwartz postula que os valores formam um contínuo de motivações distintas que se inter-relacionam. Nesse modelo são dispostas nove fatias que representam os tipos motivacionais, sendo que uma delas se subdivide entre os valores tradição e conformidade. Os lados adjacentes representam valores que compartilham metas entre si, enquanto os lados mais afastados, metas opostas ou conflitantes.

A estrutura de valores de Schwartz, disposta em formato circular, também permite observar a oposição entre os valores conflitantes de forma mais clara, já que nessa estrutura o autor propõe a organização dos tipos motivacionais em dimensões bipolares de ordem superior. Essas dimensões são: autotranscedência (universalismo e benevolência), que se contrasta com autopromoção (poder, realização e hedonismo) e abertura à mudança (hedonismo, estimulação e autodireção), que se opõe à conservação (tradição, conformidade e segurança).

O Inventário de Valores de Schwartz tem sido amplamente utilizado nas pesquisas sobre valores pessoais e transculturais. Sua primeira versão foi elaborada em 1988 e possuía originalmente 56 itens. Em 1994 foi realizada uma revisão que acabou incorporando mais uma premissa ao questionário, passando a totalizar 57 itens. No Brasil foram adicionados quatro valores característicos do país: esperteza, trabalho, sonho e vaidade, totalizando 61 itens. Esse instrumento foi validado em uma pesquisa desenvolvida por Tamayo e Schwartz (1993).

Com base na revisão da literatura, a Teoria de Valores de Schwartz foi utilizada nesse estudo para investigação das relações existentes entre os valores pessoais, a sustentabilidade e a escolha de uma organização para se trabalhar. Além da estrutura de valores básicos de Schwartz, foram consideradas para fins de análise as dimensões bipolares de ordem superior (autotranscedência, autopromoção, abertura à mudança e conservação), sendo considerada nessa pesquisa como dimensão relacionada à sustentabilidade à autotranscedência, com os valores de benevolência e universalismo que retratam o interesse por benefícios para a família, amigos e para o mundo (Axsen \& Kurani, 2013).

\subsection{Sustentabilidade}

Os impactos das mudanças climáticas e os desafios impostos pela busca do desenvolvimento sustentável têm sido amplamente debatidos pela sociedade civil, pelos governos, pelas empresas, e estudado pela academia de maneira interdisciplinar. Um dos desafios é em como reestruturar o capitalismo de forma sustentável, onde a atuação empresarial deve considerar o equilíbrio das dimensões econômica, social e ambiental, triple bottom line (TBL), nas suas decisões (Elkington, 1998; 2001). Nesse sentido, as empresas exercem papel fundamental na promoção do desenvolvimento sustentável quando consideram o TBL nas suas estratégias de negócios. 
Esse cenário leva gradativamente as empresas a considerar como parte integrante da estratégia de negócios a inclusão de metas corporativas compatíveis com o desenvolvimento sustentável. Empresas com iniciativas focadas em sustentabilidade já percebem o impacto positivo na sua produtividade, no aumento de satisfação e na retenção dos funcionários, e na contribuição com sua imagem, marca e reputação (Ryder, Shahid e Yan, 2011).

As questões e valores com foco em sustentabilidade também envolvem os indivíduos, onde o consumo consciente dos recursos naturais é uma das principais preocupações da sociedade moderna em geral (Tsarenko et al, 2013). Os valores orientados para a sustentabilidade estão relacionados com as motivações que levam um indivíduo a apresentar comportamentos percebidos e apoiados por metas de sustentabilidade. Sendo que a definição desses valores e metas se diferencia entre indivíduos, grupos sociais e culturas (Axsen \& Kurani, 2013).

Se, por um lado, valores orientados para a sustentabilidade não foram explicitados por Schwartz, por outro, o valor "proteção ambiental", que se se relaciona com sustentabilidade, mas de uma forma mais específica, pode se adequar o tipo de motivação universalismo, que corresponde com a autotranscedência e abertura para mudança. Além disso, sustentabilidade pode ser associada com benevolência e universalismo (Schwartz, 1994; Axsen \& Kurani, 2013).

\subsection{Escolha de uma organização para se trabalhar}

Os recursos humanos têm grande importância e capacidade de influenciar o desempenho organizacional, podendo representar uma fonte de vantagem competitiva sustentável (Barney \& Wright, 1998; Chadwick \& Dabu, 2009; Coff \& Kryscynski, 2011), mas, para isso, os gestores devem se atentar para a atração de talentos, a retenção dos indivíduos considerados fundamentais para a organização e sua motivação (Coff \& Kryscynski, 2011). Dessa forma, ao passo em que a atração de talentos deve ser uma das principais preocupações da alta gestão (Boswell, W.R.; Roehling, M.V.; Lepine, M.A. \& Moynihan, L.M, 2003; Coff \& Kryscynski, 2011), a escolha de um trabalho por parte de um indivíduo se torna mais relevante para as organizações.

A escolha de um trabalho é um processo que pode ser caracterizado como uma série de decisões tomadas pelo candidato a fim de saber quais trabalhos e organizações procurar para uma possível contratação e emprego (Gatewood et al, 1993). Esse processo de tomadas de decisões evidencia o livre-arbítrio que impacta no comportamento dos indivíduos a partir da forma como cada um pensa e se relaciona (Chadwick \& Dabu, 2009), tornando a escolha de uma organização para se trabalhar uma decisão complexa que, segundo Weber e Mahringer (2008), é baseada em uma coleta de informações que exercem influência no indivíduo.

Para tentar clarificar as influências no processo de escolha de uma organização para se trabalhar, segundo Boswell et al (2003), podem ser identificados 28 fatores que o autor agrupou em 14 itens de atração para um trabalho, a saber: cultura da companhia, oportunidades de crescimento, natureza do trabalho, oferta de treinamentos, balanceamento do trabalho com vida pessoal, compensação monetária, benefícios, localização, tempo de férias, nível de segurança no trabalho, tamanho da companhia, tarefas internacionais, reputação da companhia e setor de atuação.

Ainda segundo Boswell et al (2003), a cultura da organização, as oportunidades de crescimento e a natureza do trabalho possuem grande importância para os respondentes, sendo que ao serem questionados sobre quais fatores relevantes são esperados com relação às companhias empregadoras, as compensações financeiras e os benefícios, bem como a cultura da organização e a natureza do trabalho, são as variáveis mais citadas. Entretanto, o autor demonstra que novos fatores são apontados como relevantes ao longo do processo de escolha do emprego, sendo, por exemplo, a reputação da organização um item bastante lembrado no momento de se aceitar um trabalho, o que evidencia a complexidade dessa tomada de decisão.

Ao se focar nos ganhos do indivíduo apresentados por Boswell et al (2003), que são muito lembrados pelos respondentes como uma expectativa com relação às companhias empregadoras, é possível se traçar um paralelo com o conjunto de recompensas aos indivíduos abordadas por Hipólito (2002), que, segundo o autor, se dá por meio da remuneração fixa e variável, como o

Revista de Gestão Social e Ambiental - RGSA, São Paulo, v. 7, n. 3, p. 72-88, out./dez., 2013. 
salário, participação nos lucros e resultados, benefícios e programas de desenvolvimento custeados pela organização; e por meio de recompensas alternativas, como as possibilidades de mudanças de áreas e de funções dentro da organização, oportunidades de promoções, plano de carreira e carreira internacional. De maneira complementar, é possível ainda relacionar o conjunto de recompensas com a teoria dos dois fatores difundida por Frederick Herzberg, que versa sobre a satisfação e insatisfação com o trabalho por meio de fatores de higiene, como a remuneração, e de fatores de motivação, como a oportunidade de crescimento (Herzberg, Mausner \& Snyderman, 1959; GrigaLiunas \& Herzberg, 1971; Ramlall, 2004). Em um estudo que visava comparar os achados da literatura acerca da teoria dos dois fatores, Zikmund, Catalanello e Wegener (1977) concluíram que as oportunidades de crescimento (opportunity for advancement) e o salário eram os fatores que mais influenciavam a decisão de seleção de trabalho.

Já os fatores apresentados por Boswell et al (2003) referentes à empresa, que são igualmente lembrados pelos respondentes, tais como sua cultura e reputação, podem, de certa forma, se relacionar com o desempenho econômico, social e ambiental das organizações, apresentados por Elkington (1998; 2001) como TBL.

\section{MÉTODO DE PESQUISA}

Este estudo se caracteriza como uma pesquisa de campo quantitativa e descritiva, utilizandose um modelo não-experimental (Marconi \& Lakatos, 2003). A escolha do enfoque quantitativo deve-se ao fato de que os resultados de uma pesquisa quantitativa tendem a fixar com o objetivo de provar que as crenças e as proposições de um pesquisador estão próximas ou não da realidade do ambiente (Sampieri, Collado \& Lucio, 2006). Além disso, a pesquisa quantitativa permite uma medição objetiva com a quantificação dos resultados obtidos (Godoy, 1995), envolvendo a coleta e análise de dados numéricos, e aplicação de testes estatísticos (Collis \& Hussey, 2005).

O método escolhido foi o survey analítico, com o objetivo de entender se há alguma relação entre diferentes variáveis (Collis \& Hussey, 2005), a partir de uma amostra não- probabilística por conveniência, considerada pela disponibilidade dos participantes (Freitas et al, 2000) e composta por alunos que estavam cursando disciplinas na FEA/USP no primeiro semestre de 2013, em sua maioria ingressantes naquele ano. A amostra é predominantemente homogênea, pois todos os respondentes cursavam a mesma disciplina de Fundamentos da Administração, e nessa disciplina foi realizada a aplicação dos questionários. Antes da aplicação final do questionário foi realizado um pré-teste com dois estudantes voluntários para verificar seu entendimento quanto às questões.

$\mathrm{O}$ instrumento de coleta foi um questionário estruturado e impresso composto por três partes, resumidas na figura 2, e aplicado presencialmente no mês de junho de 2013 com a participação de, pelo menos, um dos pesquisadores.

\begin{tabular}{|c|c|c|}
\hline PARTE 1 & PARTE 2 & PARTE 3 \\
\hline $\begin{array}{l}\text { EMPRESAS PARA SE TRABALHAR } \\
\text { - Cartões } \\
\text { - Análise conjunta: } 81 \text { cenários, sendo } \\
9 \text { considerados representativos } \\
\text { estatisticamente (Hair Jr, J.F., Black, } \\
\text { W.C., Babin, B.J. \& Anderson, R.E., } \\
\text { 2009) } \\
\text { - Para a criação desses cenários foram } \\
\text { considerados os seguintes critérios: } \\
\text { - Desempenho econômico } \\
\text { - Desempenho socioambiental } \\
\text { - Remuneração } \\
\text { - Oportunidades de crescimento na } \\
\text { carreira }\end{array}$ & $\begin{array}{l}\text { VALORES } \\
\text { - Questionário de Perfis de } \\
\text { Valores (QVP) composto de } 40 \\
\text { assertivas que descrevem } \\
\text { objetivos e aspirações que, } \\
\text { implicitamente, apontam para a } \\
\text { importância de um tipo } \\
\text { motivacional em um indivíduo } \\
\text { (Schwartz, 2005). }\end{array}$ & $\begin{array}{l}\text { QUALIFICADORAS } \\
\text { - Questões de caráter pessoal, } \\
\text { com a finalidade de mapear o } \\
\text { perfil da amostra: } \\
\text { - sexo } \\
\text { - idade } \\
\text { - curso de graduação } \\
\text { - ... } \\
\text { - Questões relacionadas ao } \\
\text { consumo consciente (Akatu, } \\
\text { 2013). }\end{array}$ \\
\hline
\end{tabular}

Figura 2: Representação do instrumento de coleta de dados

Fonte: Elaborado pelos autores, (2013) 
A primeira parte do questionário foi estruturada com a construção de nove cenários de empresas. Esses cenários, considerados representativos estatisticamente, foram gerados a partir de 81 possíveis cenários de empresas, formulados com o apoio do software SPSS. Para a criação desses cenários, foram considerados os seguintes critérios: desempenho econômico, desempenho socioambiental, remuneração e oportunidades de crescimento na carreira.

Durante a aplicação presencial dos questionários, o quadro com os cenários das empresas foi projetado na sala de aula. Um dos objetivos de projetar os cenários foi facilitar o processo de aplicação e leitura dos participantes. Além disso, a apresentação de uma empresa de cada vez teve como objetivo diminuir o viés para o participante atribuir uma nota a cada cenário. Os nove cenários foram apresentados em razão de quatro diferentes aspectos, sendo cada um classificado como alto, médio ou baixo. Os respondentes receberam um formulário para avaliar seu interesse em trabalhar em cada uma das organizações e foram orientados a considerar as classificações de alto, médio ou baixo a partir de seus referenciais subjetivos, pois se fossem definidos parâmetros fixos, os diferentes referenciais de cada indivíduo poderiam gerar diferenças de percepção que impactariam os resultados. Para efeito de aplicação, os respondentes foram expostos às definições, construídas à luz da teoria, a saber: desempenho econômico; desempenho socioambiental; remuneração, e oportunidades de crescimento na carreira. Com isso, buscou-se construir um instrumento de coleta que fizesse um contraponto entre o desempenho da organização a partir de seu TBL incorporando a variável de desempenho socioambiental ao tradicional desempenho econômico - e as recompensas individuais, cujos fatores selecionados para avaliação foram corroborados pela literatura, indicando que os fatores mais importantes na avaliação de uma escolha de empresa são a remuneração e as oportunidades de crescimento (Zikmund, Catalanello e Wegener (1977). O objetivo era identificar a importância relativa de cada uma dessas frentes no processo de escolha da organização para se trabalhar, possibilitando aos respondentes uma tomada de decisão menos complexa com os demais fatores fixados.

A parte II do instrumento de coleta consiste no Questionário de Perfis de Valores (QVP) composto de 40 assertivas que descrevem objetivos e aspirações que, implicitamente, apontam para a importância de um tipo motivacional em um indivíduo (Schwartz, 2005). Esse instrumento já foi validado no Brasil por Tamayo e Porto (2009) e elaborado a partir do Inventário de Valores de Schwartz (1992), que se baseia na teoria dos dez tipos motivacionais categorizados pelo autor, conforme destacado na revisão da literatura.

A parte III do questionário é composta por perguntas sobre o perfil dos respondentes, considerando os dados demográficos da amostra e as atitudes de consumo consciente, com a finalidade de identificar a sensibilidade dos indivíduos com relação a alguns aspectos de socioambientais. Essas perguntas tiveram como base o Teste de Consumo do Instituto Akatu (Akatu, 2013). No entanto, diante dos resultados encontrados e o encaminhamento do estudo, optou-se por avaliar essa associação posteriormente, com uma construção mais adequada dessa problemática.

Com relação aos critérios de validação da amostra, foram excluídos oito questionários que apresentaram inconsistências. A amostra válida trabalhada na análise dos resultados foi de 203 questionários.

\section{RESULTADOS DA PESQUISA}

A amostra foi composta por estudantes de seis turmas de Fundamentos de Administração do primeiro semestre de 2013 da Faculdade de Economia, Administração e Ciência Contábeis da Universidade de São Paulo (FEA/USP), dos quais 59,6\% eram do período noturno e 40,4\%, do diurno. A amostra continha $61,6 \%$ de homens e $38,4 \%$ de mulheres. A maioria era composta por alunos de administração $(48,8 \%)$ e contabilidade $(45,3 \%)$, e apenas uma pequena parte $(5,9 \%)$ era de outros cursos. Com relação à idade, três quartos da amostra $(75,4 \%)$ tinham 20 anos ou menos, o

Revista de Gestão Social e Ambiental - RGSA, São Paulo, v. 7, n. 3, p. 72-88, out./dez., 2013. 
que é justificado por grande parte dos alunos $(93,1 \%)$ ser do primeiro ano. Condizente com esses dados, cabe destacar que $68 \%$ dos respondentes nunca trabalharam; 31,5\%, já iniciaram outra graduação; e do total, 9,4\% já haviam concluído outro curso. Cerca de metade dos respondentes $(53,3 \%)$ já fez ou faz trabalho voluntário e quase um quinto $(19,2 \%)$ já morou no exterior.

\subsection{Confiabilidade dos resultados}

De acordo com Hair et al (2009), a confiabilidade em uma pesquisa é “[...]a extensão em que uma variável ou um conjunto de variáveis é consistente com o que se pretende medir" (p. 22). Evrard et al (1997) colocam o mesmo conceito em termos mais simples ao dizer que "[...]se pudermos medir um fenômeno diversas vezes com um mesmo instrumento, devemos obter o mesmo resultado" (p. 277), associando essa ideia ao conceito de confiabilidade. Tanto Evrard et al (1997) quanto Hair et al (2009) indicam que uma das medidas de confiabilidade mais usuais é o alfa de Cronbach, medida "[...]que varia de 0 a 1 , sendo os valores de 0,60 e 0,70 considerados o limite inferior de aceitabilidade" (Hair et al, 2009, p.100). Para ter um indicador de consistência interna da pesquisa realizada, optou-se por utilizar esse indicador.

Com relação às questões de avaliação de perfil dos respondentes da parte III do questionário, associadas ao consumo consciente, o valor do alfa de Cronbach obtido foi de 0,545 , muito perto do limite de aceitabilidade de 0,6. Já para a parte II do questionário, que foi constituída pelas 40 assertivas para aferição dos valores, conforme a teoria de Schwartz (1992; 1994), o valor do alfa de Cronbach foi de 0,806 e o valor ajustado a itens padronizados, 0,812 .

O critério do Alfa de Cronbach também foi utilizado para verificar a consistência dos construtos de valores, baseados na teoria de Schwartz (1992). Nesse estudo, o objetivo foi avaliar a relação de valores associados à sustentabilidade e a escolha de uma empresa para se trabalhar. Nesse caso, interessava olhar não para os valores separadamente, mas para eles em grupos, conforme aponta Schwartz (1992). O agrupamento foi o seguinte:

- Grupo de valores Abertura à Mudança (AM): Autodireção + Estimulação + Hedonismo

- Grupo de valores Autopromoção (AP): Poder + Realização

- Grupo de valores Autotranscedência (AT): Benevolência + Universalismo

- Grupo de valores Conservação (CO): Conformidade + Segurança + Tradição

O Alfa de Cronbach obtido para o grupo de valores Abertura à Mudança (AM) foi de 0,75 e para o grupo Autopromoção (AP), 0,77. No caso do grupo Autotranscedência (AT), de 0,80 e de 0,77 para o grupo de valores Conformidade (CO). Esses valores ficaram todos acima de 0,7, referência usualmente aceita para indicar a consistência interna das medidas.

\section{ANÁLISE E DISCUSSÃO DOS RESULTADOS}

Após a consolidação da coleta de dados e a verificação da consistência interna do instrumento utilizado, apresentam-se a seguir as análises e os resultados do estudo, iniciando-se pelos resultados obtidos pela técnica da análise conjunta e, em seguida, relacionando-os aos valores sustentáveis.

\subsection{Análise conjunta}

A análise conjunta é uma metodologia estatística que tem por objetivo entender preferências individuais que compartilham uma fundamentação teórica e, ao mesmo tempo, mantém um alto grau de realismo (Hair et al, 2009). Nesse sentido, a análise conjunta permitiu a construção dos nove cenários de organizações e a análise dos dados, representando um universo de 81 cenários gerados no software SPSS, que foram todas as combinações possíveis.

O conjunto mínimo gerado pelo SPSS pelo método de geração de um conjunto de dados ortogonal e estatisticamente significativo para o objeto de estudo foi de nove cenários. É importante lembrar que o número de combinações advém da estruturação das variáveis utilizadas no trabalho. 
Como eram três níveis (alto, médio e baixo) para cada uma das quatro variáveis consideradas (Desempenho Econômico, Desempenho Socioambiental, Remuneração e Oportunidades de Crescimento), o número de cenários possíveis foi dado por $3 \times 3 \times 3 \times 3=3^{4}=81$.

Como seria inviável pedir a cada respondente avaliar os 81 cenários, optou-se pela análise conjunta, já que esta permite obter as utilidades para cada nível de cada uma das variáveis. Cada uma dessas combinações entre as variáveis é um cenário, o que, neste estudo, corresponde a uma empresa a ser avaliada pelos respondentes. Segundo Hair et al (2009, p. 360), a utilidade "[...]é o conceito mais fundamental em análise conjunta e a base conceitual para medir valor". Os autores prosseguem dizendo que "[...]a utilidade é considerada baseada no valor colocado em cada um dos níveis dos atributos". Assim, após rodar a análise conjunta, o software estatístico é capaz de calcular a utilidade de cada nível de cada variável para cada um dos respondentes. Com esses valores, é possível calcular a utilidade relativa (importância relativa) das variáveis, que foi a quantia utilizada neste estudo para investigar como as pessoas escolhem uma organização para se trabalhar.

A medida de qualidade do ajuste da análise conjunta pode ser obtida pela correlação de Pearson entre as notas atribuídas pelos respondentes e o valor calculado a partir dos coeficientes do modelo. A indicação de que o modelo utilizado foi adequado para o problema estudado veio do alto valor desse coeficiente. No total, a correlação entre todas as notas dadas pelos respondentes para as empresas e o valor ajustado do modelo foi de 0,999997. Para os respondentes, o valor mínimo foi de 0,99993 e para as empresas, de 0,999985. Esses resultados indicam somente a adequação do modelo utilizado, como um indicador de consistência da avaliação dos cenários (empresas) pelos respondentes.

O cálculo da utilidade relativa é efetuado da seguinte forma: para cada variável (Desempenho econômico, desempenho socioambiental, remuneração e oportunidades de crescimento) foi calculada, pela técnica da análise conjunta, uma utilidade para cada nível que ela pode ter (alto, médio ou baixo). Obtém-se, em seguida, o valor mínimo e o valor máximo em cada variável e calcula-se a diferença.

Seja $i$ a identificação da variável e $j$ a indicação do seu nível, temos cada utilidade como um valor $u_{i j}$, com $i$ variando de um até quatro (Desempenho Econômico, Desempenho Socioambiental, Remuneração e Oportunidades de Crescimento) e $j$ de um até três (alto, médio e baixo). Assim, a diferença (range) $R_{i}$ de cada variável é dada por:

$$
R_{\mathrm{i}}=\max \left(u_{i j}\right)-\min \left(u_{i j}\right), \quad \mathrm{j}=1,2,3
$$

A utilidade relativa de cada variável, em valor percentual, $U(\%)_{i}$ é dada por:

$$
\begin{aligned}
U(\%)_{i}= & \frac{R_{i}}{\sum_{i=1}^{4} R_{i}} \\
& \text { Nesse caso, é válida a propriedade: } \sum_{i=1}^{4} U(\%)_{i}=100 \%
\end{aligned}
$$

Tabela 1 - Importância relativa das variáveis para toda a amostra

\begin{tabular}{|l|c|}
\hline \multicolumn{1}{|c|}{ Variável } & $\begin{array}{c}\text { Importância } \\
\text { relativa }\end{array}$ \\
\hline Desempenho econômico & $16,00 \%$ \\
\hline Desempenho socioambiental & $18,13 \%$ \\
\hline Remuneração & $34,15 \%$ \\
\hline Oportunidades de crescimento & $31,72 \%$ \\
\hline
\end{tabular}

Fonte: Elaborado pelos autores (2013)

O cálculo acima é possível de ser efetuado para cada indivíduo e também para a amostra como um todo. Os valores das utilidades relativas para a amostra como um todo são vistos na Tabela 1. Pode-se notar que os fatores associados aos benefícios pessoais (salário/remuneração e oportunidades de crescimento) apresentam valores substancialmente maiores (da ordem de 30\%) do que aqueles relativos ao desempenho da organização (da ordem de 15 a 20\%). Nesse caso, a utilidade relativa atribuída à remuneração foi de $34 \%$, e para as oportunidades de crescimento, de 
$32 \%$. Com relação ao desempenho da organização, o desempenho econômico teve utilidade relativa de $16 \%$ e o socioambiental, de $18 \%$. Essa valorização maior das variáveis associadas aos benefícios individuais assemelha-se com a informação de Boswell et al (2003), que apresenta as oportunidades de crescimento e as compensações financeiras, juntamente com a cultura da companhia e especificidades do trabalho, como fatores relevantes esperados pelos respondentes com relação às companhias empregadoras.

Contudo, embora essa informação seja interessante e já permita uma validação do que Boswell et al (2003) apresentaram em comparação ao TBL das organizações, apenas encaminha uma parte da questão que este trabalho pretende responder, isto é, de que maneira valores orientados para a sustentabilidade influenciam a escolha de uma organização para se trabalhar. Nas próximas subseções foi feita a análise, de forma que fosse possível responder a questão problema dessa pesquisa.

\subsection{Utilidades relativas em função da presença dos valores}

$\mathrm{Na}$ segunda fase da análise, decidiu-se por observar como a utilidade relativa de cada variável se comporta em função da intensidade de cada grupo de valor. O primeiro passo, portanto, é observar a correlação existente entre os pares de variáveis. A Tabela 2 mostra um resumo dos valores obtidos da correlação de Pearson para cada par variável-grupo. A visualização é facilitada por meio de gráficos, apresentados na Figura 3. Cada uma das quatro variáveis (Desempenho Econômico, Desempenho Socioambiental, Remuneração e Oportunidades de Crescimento) foi cruzada com cada um dos quatro grupos de valores (AM, AP, AT e CO), lembrando-se que esses grupos, para cada indivíduo, são compostos pelas médias dos itens que compõem os valores que os integram, conforme Schwartz (1994).

Vê-se que as correlações são significativas, embora baixas $(<0,3)$, para as variáveis Desempenho Socioambiental (DE_AmbSoc) e Remuneração (BP_Salário) com relação aos grupos AP (autopromoção) e AT (autotranscedência).

Tabela 2: Correlações entre as variáveis e a intensidade de cada grupo de valores

\begin{tabular}{|l|c|c|c|c|c|c|c|c|}
\hline \multirow{2}{*}{} & \multicolumn{9}{|c|}{ Grupo } \\
\cline { 2 - 10 } & \multicolumn{2}{|c|}{ AM } & \multicolumn{2}{|c|}{ AP } & \multicolumn{2}{c|}{ AT } & \multicolumn{2}{c|}{ CO } \\
\cline { 2 - 10 } & Correl. & Sig. & Correl. & Sig. & Correl. & Sig. & Correl. & Sig. \\
\hline Desempenho econômico & $-0,014$ & 0,84 & $-0,019$ & 0,791 & $-0,013$ & 0,856 & 0,105 & 0,148 \\
\hline Desempenho socioambiental & 0,053 & 0,455 & $\mathbf{- 0 , 3 0 6}$ & $\mathbf{0 , 0 0 0}$ & $\mathbf{0 , 2 8 8}$ & $\mathbf{0 , 0 0 0}$ & $-0,064$ & 0,377 \\
\hline Remuneração & $-0,004$ & 0,950 & $\mathbf{0 , 2 0 0}$ & $\mathbf{0 , 0 0 4}$ & $\mathbf{- 0 , 1 9 8}$ & $\mathbf{0 , 0 0 5}$ & 0,002 & 0,980 \\
\hline Oport. crescimento & $-0,036$ & 0,615 & 0,081 & 0,251 & $-0,044$ & 0,538 & $-0,011$ & 0,875 \\
\hline
\end{tabular}

Fonte: Elaborado pelos autores (2013)

Para complementar essa fase, foram feitas análises de regressões envolvendo os mesmos 16 pares, resumidos na Tabela 3. Ao lado de cada regressão é apresentado o resultado ( $\boldsymbol{x}$ ou $\checkmark$ ), caso ela seja estatisticamente significativa. Os dados de qualidade do ajuste $\left(\mathrm{R}^{2}\right)$ são mostrados em cada gráfico da Figura 2. A avaliação da homogeneidade da variância dos resíduos (homocedasticidade) foi feita a partir do diagrama de dispersão dos resíduos versus valores previstos. $\mathrm{O}$ padrão encontrado foi aleatório e também não indicou a presença de ouliers. Com relação aos pontos influentes, utilizou-se, complementarmente, o critério de Cook, que mede o quanto os resíduos de todos os casos mudam se um caso particular for excluído do cálculo dos coeficientes de regressão. O maior valor encontrado para essa medida de distância foi de 0,450, abaixo do limite de 1 , acima do qual deve-se considerar a exclusão da observação.

Os dados confirmam o fato de que a maior intensidade dos grupos de valores AP e AT implica uma forma diferente de classificar as variáveis Desempenho Socioambiental e 
Remuneração. Essa conclusão é obtida a partir de todos os indivíduos que responderam a pesquisa e mostra o seguinte comportamento: quanto mais presentes são os valores de autopromoção, maior importância é atribuída à remuneração e menor importância é dada ao desempenho socioambiental da organização, refletindo a característica mais individualista traduzida por esse grupo de valores. De forma inversa, quanto maior a presença dos valores de autotranscedência, menor importância é dada à remuneração e mais importante passa a ser o desempenho socioambiental da organização.

Tabela 3: Resumo das análises de regressão envolvendo as variáveis e os grupos de valores.

\begin{tabular}{|c|c|c|c|c|c|c|c|c|}
\hline Variável & Grupo & Modelo & B & Desv. Pad. & $\beta$ & $\mathbf{t}$ & Sig & \\
\hline \multirow{8}{*}{$\begin{array}{l}\text { Desempenho } \\
\text { econômico }\end{array}$} & \multirow{2}{*}{ AM } & Const. & 0,168 & 0,030 & & 5,572 & 0,000 & \multirow{2}{*}{$x$} \\
\hline & & Regr. & $-0,002$ & 0,008 & $-0,020$ & $-0,281$ & 0,779 & \\
\hline & \multirow{2}{*}{$\mathrm{AP}$} & Const. & 0,181 & 0,028 & & 6,551 & 0,000 & \multirow{2}{*}{$x$} \\
\hline & & Regr. & $-0,006$ & 0,008 & $-0,053$ & $-0,758$ & 0,449 & \\
\hline & \multirow{2}{*}{ AT } & Const. & 0,156 & 0,027 & & 5,712 & 0,000 & \multirow{2}{*}{$x$} \\
\hline & & Regr. & 0,001 & 0,007 & 0,011 & 0,152 & 0,880 & \\
\hline & \multirow{2}{*}{$\mathrm{CO}$} & Const. & 0,131 & 0,022 & & 5,994 & 0,000 & \multirow{2}{*}{$x$} \\
\hline & & Regr. & 0,011 & 0,008 & 0,098 & 1,402 & 0,162 & \\
\hline \multirow{8}{*}{$\begin{array}{c}\text { Desempenho } \\
\text { socioambiental }\end{array}$} & \multirow{2}{*}{$\mathrm{AM}$} & Const. & 0,163 & 0,043 & & 3,821 & 0,000 & \multirow{2}{*}{$x$} \\
\hline & & Regr. & 0,005 & 0,012 & 0,030 & 0,428 & 0,669 & \\
\hline & \multirow{2}{*}{ AP } & Const. & 0,307 & 0,038 & & 8,093 & 0,000 & \multirow{2}{*}{$\checkmark$} \\
\hline & & Regr. & $-0,036$ & 0,011 & $-0,232$ & $-3,384$ & 0,001 & \\
\hline & \multirow{2}{*}{ AT } & Const. & 0,022 & 0,037 & & 0,593 & 0,554 & \multirow{2}{*}{$\checkmark$} \\
\hline & & Regr. & 0,045 & 0,010 & 0,297 & 0,411 & 0,000 & \\
\hline & \multirow{2}{*}{$\mathrm{CO}$} & Const. & 0,194 & 0,031 & & 6,271 & 0,000 & \multirow{2}{*}{$x$} \\
\hline & & Regr. & $-0,005$ & 0,011 & $-0,030$ & $-0,422$ & 0,673 & \\
\hline \multirow{8}{*}{ Remuneração } & \multirow{2}{*}{$\mathrm{AM}$} & Const. & 0,329 & 0,050 & & 6,561 & 0,000 & \multirow{2}{*}{$x$} \\
\hline & & Regr. & 0,004 & 0,014 & 0,018 & 0,258 & 0,796 & \\
\hline & \multirow{2}{*}{ AP } & Const. & 0,189 & 0,044 & & 4,254 & 0,000 & \multirow{2}{*}{$\checkmark$} \\
\hline & & Regr. & 0,044 & 0,013 & 0,240 & 3,505 & 0,001 & \\
\hline & \multirow{2}{*}{$\mathrm{AT}$} & Const. & 0,468 & 0,044 & & 10,555 & 0,000 & \multirow{2}{*}{$\checkmark$} \\
\hline & & Regr. & $-0,035$ & 0,012 & $-0,201$ & $-2,914$ & 0,004 & \\
\hline & \multirow{2}{*}{$\mathrm{CO}$} & Const. & 0,329 & 0,036 & & 9,087 & 0,000 & \multirow{2}{*}{$x$} \\
\hline & & Regr. & 0,004 & 0,013 & 0,024 & 0,343 & 0,732 & \\
\hline \multirow{8}{*}{$\begin{array}{l}\text { Oportunidades } \\
\text { de crescimento }\end{array}$} & 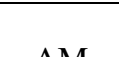 & Const. & 0,340 & 0,045 & & 7,543 & 0,000 & \\
\hline & AM & Regr. & $-0,006$ & 0,012 & $-0,036$ & $-0,505$ & 0,614 & $x$ \\
\hline & & Const. & 0,323 & 0,041 & & 7,865 & 0,000 & \\
\hline & AP & Regr. & $-0,002$ & 0,012 & $-0,011$ & $-0,154$ & 0,878 & $x$ \\
\hline & $\mathrm{T}$ & Const. & 0,354 & 0,041 & & 8,717 & 0,000 & \\
\hline & A1 & Regr. & $-0,010$ & 0,011 & $-0,065$ & $-0,925$ & 0,356 & $x$ \\
\hline & $\mathrm{CO}$ & Const. & 0,346 & 0,033 & & 10,641 & 0,000 & \\
\hline & $\mathrm{CO}$ & Regr. & $-0,011$ & 0,012 & $-0,065$ & $-0,919$ & 0,359 & $x$ \\
\hline
\end{tabular}

Fonte: Elaborado pelos autores (2013)

Como complemento às regressões feitas par a par, contendo sempre uma variável de utilidade relativa (Desempenho Econômico, Desempenho Socioambiental, Remuneração e 
Oportunidades de Crescimento) como variável dependente versus a intensidade da presença de cada um dos quatro grupos de valores (AM, AP, AT e CO) como variável independente, foi feita uma regressão múltipla para cada variável. Nesse caso, buscou-se identificar o quanto de cada variável de utilidade poderia ser explicada pelo conjunto de valores simultaneamente. Para tal, optou-se por realizar a análise de regressão pelo método stepwise, em que, a partir da explicação que cada variável oferece, o modelo a inclui ou não.

Para as variáveis Desempenho Econômico e Oportunidades de Crescimento, confirmando o resultado obtido pelas regressões par a par, não foi obtido modelo de regressão. Foram obtidos modelos lineares para as variáveis Desempenho Socioambiental e Remuneração. No caso do Desempenho Socioambiental, o modelo foi composto pelos grupos de valores autrotranscendência (AT), Autopromoção (AP) e Conservação (CO). Já para a variável Remuneração, apenas os grupos Autotranscendência (AT) e Autopromoção (AP) entraram no modelo. A Tabela 4 apresenta os resultados das regressões múltiplas. Os valores de $\mathrm{R}^{2}$ foram 0,173 e 0,322 , respectivamente. A mesma observação com relação à homogeneidade da variância dos resíduos, pontos influentes e outliers feita para as regressões simples se aplica às regressões múltiplas. O maior valor obtido para a distância de Cook foi de 0,292.

Tabela 4: Resultados das análises de regressão múltipla para as variáveis Desempenho socioambiental e Remuneração

\begin{tabular}{|l|c|c|c|c|c|}
\hline \multicolumn{7}{|c|}{ Desempenho socioambiental } \\
\hline & B & Desv. Pad. & $\boldsymbol{\beta}$ & $\mathbf{t}$ & Sig. \\
\hline Constante & 0,173 & 0,049 & & 3,501 & 0,001 \\
\hline AT & 0,057 & 0,011 & 0,383 & 5,347 & 0,000 \\
\hline AP & $-0,037$ & 0,010 & $-0,233$ & $-3,579$ & 0,000 \\
\hline CO & $-0,026$ & 0,011 & $-0,167$ & $-2,321$ & 0,021 \\
\hline \multicolumn{7}{|c|}{ Remuneração } \\
\hline \multicolumn{7}{|c|}{ Desv. Pad. } & $\boldsymbol{\beta}$ & $\mathbf{t}$ & Sig. \\
\hline Constante & 0,317 & 0,059 & & 5,379 & 0,000 \\
\hline AP & 0,046 & 0,012 & 0,252 & 3,759 & 0,000 \\
\hline AT & $-0,038$ & 0,012 & $-0,215$ & $-3,213$ & 0,002 \\
\hline
\end{tabular}

Fonte: Elaborado pelos autores

De maneira geral, o resultado observado pelas regressões simples foi confirmado. O comportamento inverso da influência dos grupos de valores autotranscendência e autopromoção é facilmente verificado pelo sinal dos coeficientes do modelo e sua importância é confirmada pelos valores de beta $(\beta)$. A única diferença é a presença do grupo conservação na variável Desempenho Socioambiental (com nível de significância um pouco mais alto que os demais, de 2,1\%, mas menor que os 5\% adotados). Sua presença indica que quanto mais intensa a presença do grupo conservação para o indivíduo, menor importância será atribuída ao Desempenho Socioambiental da organização. Isso significa que, isoladamente, esse grupo de valores não contribui para a avaliação dessa variável, mas em conjunto com as demais pode ter relevância. Estudos subsequentes utilizando metodologia similar serão necessários para esclarecer essa questão.

\subsection{Consolidação das análises: valores sustentáveis e a escolha das organizações}

Com a análise estatística dos dados, bem como com a revisão teórica, é possível responder ao problema proposto por esta pesquisa que era "qual a influência dos valores voltados para a sustentabilidade na escolha de uma organização para se trabalhar?".

Conforme a discussão acima, ficou claro que há diferenças entre como as pessoas enxergam as organizações e as escolhem como opção para se trabalhar em razão dos valores pessoais. Os 
indícios são de que quanto maior a presença da autotranscedência (AT), que contém os valores ligados às questões de sustentabilidade, conforme relatado por Axsen e Kurani (2013), maior importância é atribuída ao desempenho socioambiental da organização em contraponto à remuneração individual e ao fator de higiene (Herzberg, Mausner \& Snyderman, 1959; Grigaliunas \& Herzberg, 1971; Ramlall, 2004) e apresentada pelos trabalhos de Boswell et al (2003) como relevante no processo de escolha de um trabalho. O comportamento inverso é observado na presença de valores de autopromoção (AP), que, de acordo com a teoria de Schwartz, constituem o grupo oposto ao de autotranscedência (AT). A Figura 3 resume o comportamento encontrado em nosso estudo, a partir dos diagramas de dispersão vistos na Figura 4.

Mais do que isso, considerando que uma organização sustentável é aquela que alcança o equilíbrio e o bom desempenho entre as dimensões econômica, social e ambiental (Elkington, 2001) e, como há indiferença independentemente da intensidade da presença dos grupos de valores com relação ao desempenho econômico, sendo que a variação da escolha só aparece no desempenho socioambiental, percebe-se um alinhamento entre valores pessoais sustentáveis e escolha por uma organização sustentável.

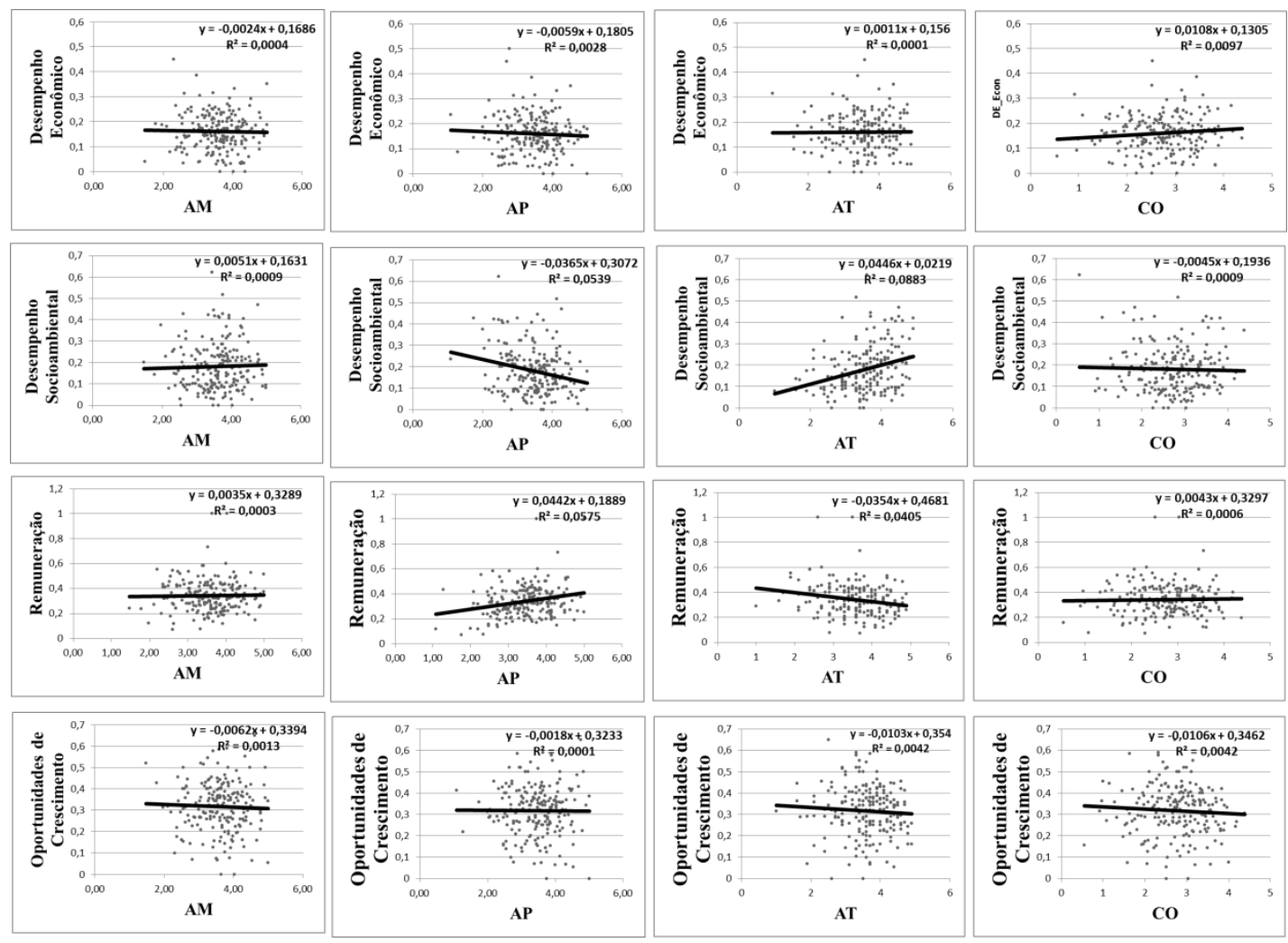

Figura 3: Diagramas de dispersão para as quatro variáveis (ordenada) com relação a intensidade de cada um

Fonte: Elaborado pelos autores (2013) dos grupos de valores (abscissas) 


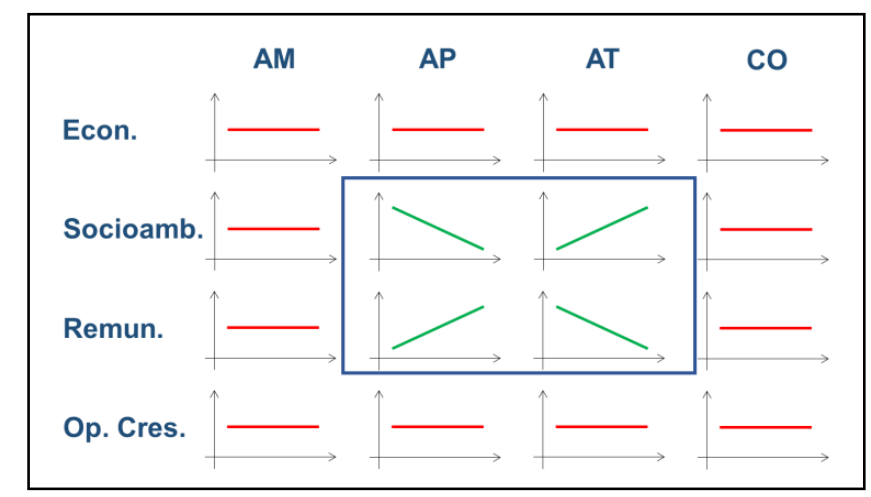

Figura 4: Resumo do comportamento encontrado no estudo: a presença ou ausência de valores sustentáveis influencia o tipo de organização escolhida para se trabalhar.

Fonte: Elaborado pelos autores (2013)

\section{CONSIDERAÇÕES FINAIS}

De maneira geral, essa pesquisa possibilitou testar e validar o modelo metodológico proposto para esse estudo, sendo que foi possível responder a todos os objetivos propostos inicialmente. A análise dos dados identificou indícios de que quanto maior a presença da dimensão autotranscedência - que compreende os valores universalismo e benevolência (Schwartz, 1992) do indivíduo, maior a importância do desempenho socioambiental da organização, e, portanto, da sustentabilidade (Elkington, 1998; 2001), em contraponto à remuneração individual (Hipólito, 2002; Boswell et al, 2003). Foram encontradas diferenças estatisticamente significativas para as variáveis desempenho socioambiental e remuneração. Assim, quanto maior a intensidade dos valores do grupo autotranscendência, maior a importância do desempenho socioambiental e menor a importância da remuneração. O comportamento oposto é observado pelo grupo autopromoção.

Nesse sentido, reafirma-se o que Axsen e Kurani (2013) encontraram em seu estudo, baseado na estrutura de valores básicos de Schwartz (1992), ou seja, a dimensão autotranscedência, constituída pelos valores benevolência e universalismo que retratam o interesse por benefícios para a família, amigos e para o mundo, relaciona-se com a sustentabilidade. Assim, há indícios de que a escolha de uma organização para se trabalhar é afetada pela intensidade dos valores orientados à sustentabilidade, de forma que quanto mais presentes esses valores mais importante é o desempenho sustentável da organização.

Este estudo mostra que os valores pessoais podem ser importantes para escolha de organizações para se trabalhar e devem ser levados em conta tanto por empregadores quanto por candidatos a posições. Do ponto de vista das contribuições para a academia, destaca-se que o modelo teórico desenvolvido, partindo de um sólido referencial teórico para determinar as variáveis, atrelado a um instrumento de coleta já validado, possibilita a replicação em estudos comparativos com foco em experimentos e o aprofundamento das análises das variáveis. Além disso, os resultados contribuem com a escassa literatura que analisa concomitantemente as dimensões valores pessoais orientados para a sustentabilidade e a escolha de uma organização para se trabalhar.

Os executivos e gestores, por sua vez, têm como referência os resultados de um estudo empírico da influência da sustentabilidade nos valores pessoais, e que apresenta indícios de influenciar na escolha de uma organização para se trabalhar. Nesse sentido, este estudo contribui para discussões sobre estratégias corporativas e programa de atração de talentos mais adequados ao perfil de valores desejado de seus colaboradores, destacando ainda a relevância do desempenho socioambiental para determinados grupos.

\subsection{Limitações do estudo}

Durante o processo de análise dos dados foram identificados limites do estudo, a saber: amostra homogênea e não-estatisticamente representativa; viés de ordem fixa da apresentação dos 
nove cartões com os cenários das organizações, na coleta dos dados; pesquisa com baixa confiabilidade, pois a amostra foi pequena, e considerada homogênea pela idade e pelo nível de escolaridade (Tamayo \& Porto, 2009); análise conjunta não permitiu calcular o desvio-padrão com os nove cenários gerados das organizações, pois não houve graus de liberdade suficientes para computar desvios-padrão para as utilidades em cada uma dos quatro critérios (econômico, socioambiental, remuneração e oportunidades de crescimento), devido a ausência de holdouts, não previstos na geração de um conjunto ortogonal de dados pela análise conjunta, e por fim, a utilização do questionário resumido de Schwartz com 40 questões, que já havia sido validado com limitações por Tamayo e Porto (2009). Além disso, o questionário força o agrupamento e a avaliação de variáveis diferentes em uma mesma premissa. A teoria já apontava esse limite, o que levou a análise a ser baseada nos grupos de valores (dimensões bipolares de ordem superior de Schwartz).

\subsection{Recomendações para pesquisas futuras}

Considerando que a metodologia proposta foi validada, é recomendável que seja feita a aplicação de outras pesquisas no formato eletrônico, possibilitando a eliminação do viés da ordem fixa dos cartões e um maior alcance, aumento e diversidade da amostra. Além disso, a replicação do estudo com estudantes de outras faculdades, profissões e diferentes níveis de escolaridade poderão ser agrupadas para fins de comparação das amostras. É recomendável que seja considerado uma quantidade maior de cenários das organizações na aplicação dos questionários para que seja possível calcular o desvio-padrão.

As ideias presentes neste estudo podem subsidiar outras pesquisas nas quais sejam usados diferentes referenciais de valores, como o de Rokeach (1973), por exemplo. Além disso, podem ser construídos instrumentos que avaliem outros aspectos adicionais à escolha de uma organização para se trabalhar, por exemplo, com a inclusão de elementos de retenção ou de desenvolvimento dos funcionários e buscar-se relações com os valores pessoais.

\section{REFERÊNCIAS}

Allport, G. W. (1961) Pattern and growth in personality. New York: Holt, Rinehart \& Winston.

Axsen, J. \& Kurani, K. S. (2013) Developing sustainability-oriented values: Insights from households in a trial of plug-in hybrid electric vehicles. Global Environmental Change 23, 70-80.

Barney, J. B. \& Wright, P. M. (1998) On becoming a strategic partner: The role of human resources in gaining competitive advantage. Human Resource Management, 37, 31-46.

Boswell, W.R., Roehling, M.V., Lepine, M.A. \& Moynihan, L.M. (2003) Individual job-choice decisions and the impact of job attributes and recruitment practices: a longitudinal field study. Human Resource Management, 42(1), 23-37.

Chadwick, C. \& Dabu, A. (2009) Human resource, human resource management, and the competitive advantage of firms: toward a more comprehensive model of casual linkage. Organization Science, 20(1), 253-272.

Coff, R. \& Kryscynski, D. (2011) Drilling for micro-foundations of human capital-based competitive advantages. Journal of Management, 3(5), 1429-1443, Set.

Collis, J. \& Hussey, R. (2005) Pesquisa em administração: um guia prático para alunos de graduação e pós-graduação. (2 ${ }^{\mathrm{a}}$ ed.) Porto Alegre: Bookman.

Edelman. (2012) Goodpurpose: executive summary. Edelman Goodpurpose. Recuperado em: 21 jul.2013, de http://www.scribd.com/doc/90411623/Executive-Summary-2012-Edelmangoodpurpose $\%$ C2\%AE-Study.

Elkington, J. (1998) Partnerships from cannibals with forks: the triple bottom line of 21st-century business. Environmental Quality Management, 8(1), 37-51.

Elkington, J. (2001) Canibais com garfo e faca. Makron Books. 
Evrard, Y., Pras, B. \& Roux, E. (1997) Market études et recherches en marketing : fondements, méthodes. en collaboration avec Jean-Marie Choffray, Anne-Marie Dussaix, Michel Claessens, Paris: Nathan.

Freitas, H., Oliveira, M., Saccol, A.Z., Moscarola, J. (2000) O método de pesquisa survey. Revista de Administração da USP - RAUSP, 35(3), 105-112.

Gatewood, R.D., Gowan, M.A. \& Lautenschlager, G.J. (1993) Corporate image, recruitment image, and initial job choice decisions. The Academy of Management Journal, 36(2), 414-27.

Godoy, A. S. (1995) Introdução à pesquisa qualitativa e suas possibilidades - Revista de Administração de Empresas, 35(2), 57-63.

Grigaliunas, B. S. \& Herzberg, F. (1971) Relevancy in the test of motivator-hygiene theory. Journal of Applied Psychology. 55(1), 73-79.

Hair Jr, J.F., Black, W.C., Babin, B.J. \& Anderson, R.E. (2009) Análise multivariada de dados, (6 ${ }^{\mathrm{a}}$ ed.), Porto Alegre: Bookman.

Hart, S.L. \& Milstein, M. B. (2004) Criando valor sustentável. RAE Executivo, 3(2).

Herzberg, F., Mausner, B. \& Snyderman, B. (1959) The motivation to work, Nova York: John Wiley.

Hipólito, J. A. M. (2002) Sistema de recompensas: uma abordagem atual. In Fleury, M. T. L. (coord.) et al. As pessoas na organização ( $\left.5^{\mathrm{a}} \mathrm{ed}\right)$ São Paulo: Editora Gente.

May, P. H., Lustosa, M. C. \& Vinha, V. (Orgs.)(2003) Economia do meio ambiente: teoria e prática. Rio de Janeiro: Elsevier.

Marconi, M.A. \& Lakatos, E. M. (2003) Fundamentos da metodologia científica. (5 ${ }^{\text {a }}$ ed.) São Paulo: Atlas.

Pato, C. M. L. (2004) Comportamento ecológico: relações com valores pessoais e crenças ambientais, Tese (doutorado) - Instituto de Psicologia - Universidade de Brasília.

Ramlall, S. A (2004) Review of employee motivation theories and their implications for em-ployee retention and within organizations. The Journal of American Academy of Business, Cambridge, September.

Ryder, G., Shahid, F., Yan, S.(2011) The grid intelligent planning framework: planning electric utility investments in a time of accelerating change. In: Design, user experience, and usability. theory, methods, tools and practice. Springer Berlin Heidelberg.

Rokeach, M. (1973) The nature of human values. New York: Free Press.

Sampieri, R.H., Collado, C.F. \& Lucio, P.B. (2006) Metodologia de pesquisa. (3 ${ }^{a}$ ed.) São Paulo: McGraw-Hill.

Schwartz, S. H. (1992) Universals in the content and structure of values: Theoretical advances and empirical tests in 20 countries. Advances in experimental social psychology, 25, 1- 64

Schwartz, S. H. (1994) Are there universal aspects in the structure and contents of human values? Journal of Social Issues, 50 (4), 19- 45.

Schwartz, S. H. (1996) Value priorities and behavior: applying a theory of integrated value systems. In: The psychology of values: the ontario symposium. Ontario e New Jersey: Mahwah, 53-75.

Schwartz, S. H. (1999) A theory of cultural values and some implications for work. Applied psychology, 48(1), 23-47.

Schwartz, S. H. (2005) Valores humanos básicos: seu contexto e estrutura intercultural. In: Tamayo, A., Porto, J. (Orgs.) Valores e comportamentos nas organizações. São Paulo: Vozes.

Schwartz, S. H., \& Bardi, A. (2001) Value hierarchies across cultures: taking a similarities perspective. Journal of Cross Cultural Psychology, 32, 268-290.

Schwartz, S. H., \& Bilsky, W. (1987) Toward a psychological structure of human values. Journal of Personality and Social Psychology, 53, 550-562.

Sutherland, M. M., Torricelli, D. G., \& Karg, R. F. (2002) Employer-of-choice branding for knowledge workers. S Afr J of Business Manage, 33(4), 13-20.

Tamayo, A., Lima, A., Marques, J. \& Martins, L. (2001) Prioridades axiológicas e uso de preservativo. Psicologia: reflexão e crítica, Porto Alegre, 4(1), 167-175. 
Tamayo, A. \& Schwartz, S. H. (1993) Estrutura motivacional dos valores. Psicologia: teoria e pesquisa, 9, 329-348.

Tamayo, A. \& Porto, J. (2009) Validação do questionário de perfis de valores (QPV) no Brasil. Psicologia: Teoria e Pesquisa, 25(3), 369-376

Tsarenko, Y., Ferraro, C., Sands, S. \& Mcleod, C. (2013) Environmentally conscious consumption: the role of retailers and peers as external influences. Journal of Retailing and Consumer Services, 20(3), 302-310.

Weber, A. \& Mahringer, H. (2008) Choice and success of job search methods, Empirical Economics, 35, 153-178.

Zikmund, W. G., Catalanello, R. F. \& Wegener, S. M. (1977) The accounting student's job-rating criteria: an experiment, 11 (3), 729-735.

Data da submissão: 23/09/2013

Data da publicação: 16/12/2013 